Editorial

\title{
Acknowledgment to Reviewers of Antibodies in 2020
}

\section{Antibodies Editorial Office}

Citation: Citation: Antibodies

Editorial Office. Acknowledgment to Reviewers of Antibodies in 2020.

Antibodies 2021, 10, 5. https://doi.org/ 10.3390/antib10010005

Published: 25 January 2021

Publisher's Note: MDPI stays neutral with regard to jurisdictional claims in published maps and institutional affiliations.

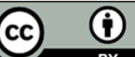

Copyright: $\odot 2021$ by the author. Licensee MDPI, Basel, Switzerland. This article is an open access article distributed under the terms and conditions of the Creative Commons Attribution (CC BY) license (http://creativecommons.org/licenses/by/4.0/).

MDPI AG, St. Alban-Anlage 66, 4052 Basel, Switzerland

Peer review is the driving force of journal development, and reviewers are gatekeepers who ensure that Antibodies maintains its standards for the high quality of its published papers. Thanks to the cooperation of our reviewers, in 2020, the median time to first decision was 17 days and the median time to publication was 59.5 days. The editors would like to express their sincere gratitude to the following reviewers for their precious time and dedication, regardless of whether the papers were finally published:

Adolf-Bryfogle, Jared

Åhlin, Anders

Alam, Md Kausar

Albarnaz, Jonas D.

Al-Saleem, Fetweh

Alt, Karen

Angkawidjaja, Clement

Antigua, Khristine Joy C.

Arunkumar, Abhiram

Axiotis, Evangelos

Azam, Aa Haeruman

Ban, Bhupal

Barfield, Robyn

Bawadekar, Mandar

Bax, Heather

Bernard, Nicole

Berrondo, Monica

Berthelot, Laureline

Bianchini, Rodolfo

Blot-Chabaud, Marcel

Boyd, Andrew

Breitling, Frank

Brooks, Cory L.

Bulatov, Emil

Challa, Dilip

Chen, Long

Chen, Shih-Chieh

Cheng, Luisa W.

Dahiya, Satinder

Danilov, Sergei M.

Dar, Mohd Saleem

Davey, Martin

De Kruif, John

Debadyuti, Ghosh

Dessain, Scott K.

\author{
Devasundaram, Santhi \\ Devaurs, Didier \\ Dixit, Surjit \\ Duranti, Claudia \\ Eason, James \\ Elvington, Michelle \\ Escobar-Cabrera, Eric \\ Fuentes-Lopez, Sandra \\ Gallagher, D. Travis \\ Gan, Samuel \\ Ganguly, Samit \\ Garcia Parra, Jezabel \\ Gilburd, Boris \\ Golay, Josee \\ Gorovits, Boris \\ Gorshkova, Ekaterina N. \\ Gosav, Evelina Maria \\ Gottschalk, Stephen \\ Goulet, Dennis R. \\ Grubić Kezele, Tanja \\ Hadži, San \\ Halkias, Joanna \\ Hamilton, Robert G. \\ Hammers, Christoph M. \\ Hatano, Yuichiro \\ Hazlett, Karsten \\ Healey, Gareth \\ Hiruta, Yuki \\ Hoebeke, Johan \\ Homma, Takujiro \\ Houen, Gunnar \\ Hwang, Dobeen \\ Hytti, Maria \\ Iaccino, Enrico \\ Idda, Maria Laura
}


Ippolito, Gregory

Jakobsson, Eric

Jayaraman, Sundararajan

Jeliazkov, Jeliazko R.

Johnson, Dylan M.

Jones, Martina

Joshi, Kamal

Kalra, Raj Singh

Kang, Min-Jung

Karsten, Christina Beatrice

Kazemian, Majid

Khan, Naeem

Khatun, Amina

Kolawole, Abimbola

Korona, Dagmara

Kowal, Krzysztof

Krause, Frank

Lamichhane, Purushottam

Lee, Sukmook

Leyton, Jeffrey V.

Li, Tommy

Lima, Wanessa C.

Liou, Chian-Jiun

Liu, Xianglei

Lo, Shyh-Chyi

Lopalco, Lucia

Lub-de Hooge, Marjolijn N.

Lucas, Andrew T.

Ma, Buyong

Mack, Matthias

Maglio, Ornella

Manez, Rafael

Manna, Saikat

Mansour, Salah

Martin, Andrew C. R.

Martins, Sonia

Matsushita, Shuzo

Mazor, Ohad

Mehta, Paras

Mo, Fei

Mukherjee, Shibabrata

Mukherjee, Somnath

Nemenoff, Raphael

Nesterov-Mueller, Alexander

Nicoletti, Loredana

Nijhof, Inger

Nishikimi, Akihiko

Nordenfelt, Pontus

Oh, Eun-Jee

Onaca, Nicholas

Ortiz, Andric
Ozen, Mehmet

Pasternak, Gerard

Patil, Veerupaxagouda

Pellefigues, Christophe

Pezzoni, Giulia

Plans-Rubi, Pedro

Poole, Brian D.

Profumo, Elisabetta

Qi, Junpeng

Rader, Christoph

Ravichandran, Supriya

Reddy, Sai T.

Reuter, Stefan

Rieder, Michael

Riley, Lee

Rispens, Theo

Rokstad, Anne Mari

Roumenina, Lubka

Rubin, Robert L.

Russo, Giulio

Sasaki, Motoko

Scheu, Stefanie

Schlothauer, Tilman

Schorzman, Allison N.

Schrum, Adam

Scott, Andrew

Sharma, Preeti

Shim, Hyunbo

Sochaj-Gregorczyk, Alicja Maria

Spoerl, David

Stang, Espen

Strasser, Richard

Sun, Wei

Sutton, Brian

Takagi, Motoki

Tanha, Jamshid

Teplyakov, Alexey

Thaker, Youg Raj

Theise, Neil

Tsuchikama, Kyoji

Vance, David

Varki, Nissi M.

Vartak, Abhishek

Vattepu, Ravi

Velikova, Tsvetelina

Venkatesan, Thiagarajan

Vidarsson, Gestur

Wagner, Alain

Wang, Shaobin

Weitkamp, Hendrik

Wheatley, Adam 
Williamson, E. Diane

$\mathrm{Wu}$, Yee Ling

Xiao, Yongsheng

Yamaki, Kouya

Yang, Kun

Yin, Tianlei

$\mathrm{Yu}$, Chunsong
Zagożdżon, Radosław

Zhang, Rui

Zhang, Weiping

Zhang, Xiangmin

Zhou, Qun

Zhou, Weijie

Zielonka, Stefan 